\title{
VASODILATION INCREASES PULSE PRESSURE VARIATION, MIMICKING HYPOVOLEMIC STATUS IN RABBITS
}

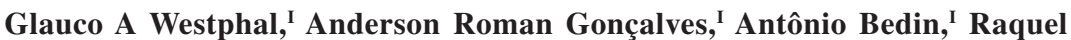 \\ Bissacotti Steglich, ${ }^{\text {I }}$ Eliezer Silva, II Luiz Francisco Poli-de-FigueiredoII
}

doi: $10.1590 / \mathrm{S1807-59322010000200011}$

Westphal GA, Gonçalves AR, Bedin A, Steglich RB, Silva E, Poli-de-Figueiredo LF. Vasodilation increases pulse pressure variation, mimicking hypovolemic status in rabbits. Clinics. 2010;65(2):189-94.

OBJECTIVE: To test the hypothesis that pulse pressure respiratory variation (PPV) amplification, observed in hypovolemia, can also be observed during sodium nitroprusside (SNP)-induced vasodilation.

INTRODUCTION: PPV is largely used for early identification of cardiac responsiveness, especially when hypovolemia is suspected. PPV results from respiratory variation in transpulmonary blood flow and reflects the left ventricular preload variations during respiratory cycles. Any factor that decreases left ventricular preload can be associated with PPV amplification, as seen in hypovolemia. METHODS: Ten anesthetized and mechanically ventilated rabbits underwent progressive hypotension by either controlled hemorrhage (Group 1) or intravenous SNP infusion (Group 2). Animals in Group $1(\mathrm{n}=5)$ had graded hemorrhage induced at $10 \%$ steps until 50\% of the total volume was bled. Mean arterial pressure (MAP) steps were registered and assumed as pressure targets to be reached in Group 2. Group $2(n=5)$ was subjected to a progressive SNP infusion to reach similar pressure targets as those defined in Group 1. Heart rate (HR), systolic pressure variation (SPV) and PPV were measured at each MAP step, and the values were compared between the groups.

RESULTS: SPV and PPV were similar between the experimental models in all steps ( $\mathrm{p}>0.16$ ). SPV increased earlier in Group 2. CONCLUSION: Both pharmacologic vasodilation and graded hemorrhage induced PPV amplification similar to that observed in hypovolemia, reinforcing the idea that amplified arterial pressure variation does not necessarily represent hypovolemic status but rather potential cardiovascular responsiveness to fluid infusion.

KEYWORDS: Volume replacement; hemodynamics; hemorrhagic shock; cardiac function; vasodilation.

\section{INTRODUCTION}

Many critically ill patients are hemodynamically unstable. This instability may be caused by cardiac dysfunction, vasodilation, hypovolemia or the association of these factors. Independent of hemodynamic behavior, patients are subjected to volume infusion or withdrawal at

\footnotetext{
I Department of Medicine, Universidade da Região de Joinville (Univille) Joinville/SC, Brazil

II Intensive Care Unit, Hospital Israelita Albert Einstein - São Paulo/SP, Brazil

III Department of Surgery, Faculdade de Medicina da Universidade de São - São Paulo/SP, Brazil

Email: glauco.w@brturbo.com.br

Tel.: 5547 3423.0304

Received for publication on August 05, 2009

Accepted for publication on November 03, 2009
}

some point during their hospitalization..$^{-4}$ In these situations, the correct and early definition of intravascular content is essential to avoid tissue ischemia, resulting in multiple organ dysfunction. ${ }^{4-6}$ Therefore, the correct diagnosis of fluid status must be accurately and rapidly achieved to avoid both hypoperfusion related to volume depletion and fluid overload followed by unnecessary infusion. Hence, accurate hemodynamic monitoring methods are necessary to help us to perform fluid management. ${ }^{4,7,8}$

Pulse pressure respiratory variation (PPV) has generally been demonstrated to be much more accurate for the detection of fluid responsiveness than cardiac filling pressures. $^{9-12}$ The former method is also more reliable than other dynamic parameters, such as systolic pressure respiratory variation (SPV) or stroke volume respiratory variation (SVV), and it can recognize volume contraction 
earlier than the others. ${ }^{9,10,13}$ Hence, PPV has become largely recommended to guide volume expansion in patients with hemodynamic instability. ${ }^{9-12}$

We have recently reported pulse oximetry wave variation as a noninvasive tool to assess volume status in cardiac surgery ${ }^{14}$. However, positive pressure ventilation promotes cyclical changes in stroke volume (SV), and is coupled with arterial pulse pressure changes. SV and arterial pulse pressure rise during inspiration. Conversely, SV comes down during expiration. PPV is directly influenced by the $\mathrm{SV}$ and is mathematically expressed as the percentage of the variation between systolic and diastolic pulse pressure obtained in a single ventilatory cycle. Patients with low PPV are insensitive to cyclic changes in preload induced by mechanical ventilation, and they operate on the flat portion of the Frank-Starling curve (non-responders). On the other hand, when PPV is high, patients are operating on the steep portion of the Frank-Starling curve and can respond with an increase in SV after fluid infusion (responders). ${ }^{9,13,15,16}$

However, the method has some limitations, and they need to be accounted for. The magnitude of PPV variations can be influenced not only by the cardiovascular responsivity status but can also be related to spontaneous breathing, arrhythmia, tidal volume above $10 \mathrm{ml} / \mathrm{kg}$ or below $8 \mathrm{ml} / \mathrm{kg}$, right ventricular dysfunction, pulmonary arterial hypertension, intrinsic or extrinsic positive end-expiratory pressure (PEEP), and alterations in arterial wall stiffness such as the one which occurs with vasodilation. ${ }^{9,16,17}$

Pizov et al. (1988) observed that both absolute (hemorrhagic shock) and relative (pharmacologic vasodilation) hypovolemia are related to the amplification of SPV in anesthetized dogs. They observed that, under absolute hypovolemic conditions, SPV is greater when compared with SPV during pharmacological vasodilation. ${ }^{18}$ More recent publications found that PPV has greater accuracy than SPV at evaluating cardiovascular responsivity in critically ill patients. ${ }^{9,11,12}$

These data show the relevance of clinical knowledge about the behavior of PPV during vasodilation. Hence, comparing PPV behavior in hypovolemic conditions and under vasodilation may be useful in clinical settings.

The purpose of this study is to compare the effects of hypovolemia and pharmacological vasodilation on arterial pressure traces.

\section{MATERIALS AND METHODS}

\section{Study design and animal preparation}

This experimental, observational and comparative study was performed at the Experimental Surgical Center.
The study was approved by an Institutional Research Consulting Board. Twenty-one New Zealand male rabbits $(1.6 \mathrm{~kg} \pm 0.3 \mathrm{~kg})$ were arbitrarily divided into two groups. Animals in Group $1(\mathrm{n}=5)$ had induced hemorrhage, and Group $2(\mathrm{n}=5)$ had induced pharmacologic hypotension with a SNP infusion. Five rabbits were used in a pilot study, and six rabbits died before the conclusion of the experiment. The animals were fasted for $12 \mathrm{~h}$ before the start of the experiment, and free access to water was allowed.

The animals received subcutaneous pre-anesthetic medication (ketamine $15 \mathrm{mg} / \mathrm{kg}$, acepromazine $1 \mathrm{mg} / \mathrm{kg}$ and dexamethasone $150 \mu \mathrm{g} / \mathrm{kg}$ ), the auricular vein was cannulated with a 24 -gauge catheter and a $0.9 \% \mathrm{NaCl}$ solution was started at $5 \mathrm{~mL} / \mathrm{kg} / \mathrm{h}$. The rabbits were placed in the supine position. General anesthesia was started and maintained with venous ketamine $(2 \mathrm{mg} / \mathrm{kg})$ and $1 \%$ inhaled halothane with oxygen flow at $2 \mathrm{~L} / \mathrm{min}$.

After a tracheotomy was performed, an endotracheal tube was inserted midway along the trachea, and controlled mechanical ventilation was started with a neonatal ventilator (Bear BP-200, Bear medical systems, Inc., Riverside, CA, USA). The ventilation settings were: tidal volume of $15 \mathrm{~mL} /$ $\mathrm{kg}$, respiratory frequency of $25 \mathrm{rpm}$, fractional concentration of oxygen $\left(\mathrm{FiO}_{2}\right)$ at $100 \%$ and PEEP of $3 \mathrm{~cm} \mathrm{H}_{2} \mathrm{O}$, resulting in a peak airway pressure between 10 and $15 \mathrm{~cm} \mathrm{H}_{2} \mathrm{O}$.

The right carotid and internal jugular vein were cannulated with 22-gauge polyethylene catheters to monitor arterial pressure and fluid administration and to induce the controlled hemorrhage. In order to replace any deficits that may have taken place while fasting prior to the experiment, rabbits were infused with $4 \mathrm{~mL} / \mathrm{kg}$ of $0.9 \% \mathrm{NaCl}$. After 30 min of cardiovascular stabilization, the hemorrhagic shock was initiated.

The arterial catheter was connected to a pressure transducer (DTX Plus DT-4812NA, Becton \& Dickinson, Franklin Lakes, NJ, USA) that was linked to a multiparametric monitor (S/5 Light-Solo ${ }^{\circledR}$, Datex-Ohmeda, Helsinki, Finlândia) to record continuous heart rate (HR) and digital arterial pressure. These parameters were transferred to a personal computer with $\mathrm{S} / 5$ Collect $^{\circledR}$ (Datex-Ohmeda, Helsinki, Finlândia) software.

\section{Experimental protocol}

Experimental procedures were performed as follows. Controlled hemorrhagic shock (Group 1): after baseline (T0) data recording (HR, arterial pressure, SPV and PPV), manual blood withdrawal through the central venous catheter was performed with steps of $10 \%$ of the estimated volemia $(80 \mathrm{~mL} / \mathrm{kg})$ at a rate of $5 \mathrm{~mL} /$ minute, until $50 \%$ of 
the total volume was bled. At each bleeding step (T1-10\%, $\mathrm{T} 2-20 \%, \mathrm{~T} 3-30 \%, \mathrm{~T} 4-40 \%$ and $\mathrm{T} 5-50 \%$ ), hemodynamic data were digitally recorded again. Pharmacologic hypotension (Group 2): the mean values of mean arterial pressure (MAP) obtained at each of the bleeding steps observed in Group 1 were defined as the target to be reached with SNP infusion. The SNP infusions were started at $0.5 \mu \mathrm{g} / \mathrm{kg} / \mathrm{min}$. Progressive SNP dose increments were performed until the MAP targets were reached. Hemodynamic data were digitally recorded at each induced hypotension step.

The pressure waveforms were analyzed off-line on a personal computer. We identified the arterial pressure respiratory variation and the systolic and diastolic pressures (Figure 1). In each respiratory cycle, systolic pressure and pulse pressure during inspiration $\left(\mathrm{Ps}_{\max }\right.$ and $\left.\mathrm{Pp}_{\max }\right)$ and expiration $\left(\mathrm{Ps}_{\min }\right.$ and $\mathrm{Pp}_{\min }$ ) were identified. Respiratory changes in SPV and PPV (in \%) were calculated using the following formulas ${ }^{10}$ :

$\operatorname{SPV}(\%)=100 x\left(\mathrm{Ps}_{\max }-\mathrm{Ps}_{\text {min }}\right) /\left[\left(\mathrm{Ps}_{\max }+\mathrm{Ps}_{\min }\right) / 2\right] ;$

$\operatorname{PPV}(\%)=100 \times\left(\mathrm{Pp}_{\max }-\mathrm{Pp}_{\min }\right) /\left[\left(\mathrm{Pp}_{\max }+\mathrm{Pp}_{\min }\right) / 2\right]$

After the experiments, all animals were euthanized by intravenous pentobarbital overdose and hypertonic potassium solution.

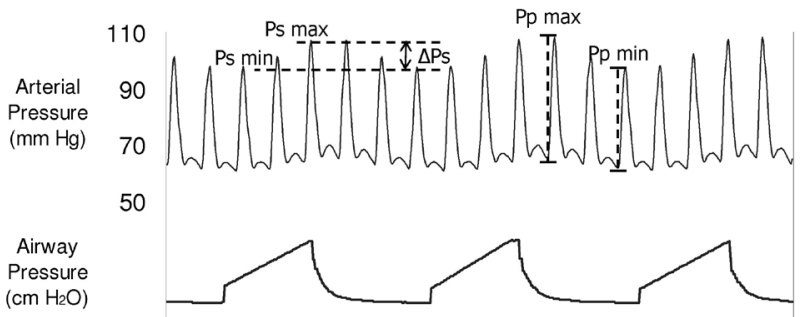

Figure 1 - The representative trace shows the arterial pressure fluctuations during mechanical ventilation. The systolic pressure is maximal during inspiration and declines in expiration. Similarly, arterial pulse pressure is maximal during inspiration and minimal during expiration. $\mathrm{Pp}_{\max }$, maximal arterial pulse pressure; $\mathrm{Pp}_{\min }$, minimal arterial pulse pressure; $\mathrm{Ps}_{\max }$, maximal arterial systolic pressure; $\mathrm{Ps}_{\min }$, minimal arterial systolic pressure.

\section{Statistical methodology}

For the statistical analysis, NCSS: Statistical Software 2000 and PASS 2000: Power Analysis \& Sample Size were used. The results were expressed as the mean \pm standard error of mean (SEM). Data were compared using Student's $t$-test for continuous variables. Mixed ANOVA was conducted to analyze repeated measures between group interactions. All tests were two-tailed, and a $P$ value $<0.05$ was considered statistically significant.

\section{RESULTS}

All animals were hemodynamically stable at baseline (BL). Animal weight ranged from $1.3 \mathrm{~kg}$ to $1.9 \mathrm{~kg}$. The estimated average volemia $(80 \mathrm{~mL} / \mathrm{kg})$ ranged from 104 $\mathrm{mL}$ to $152 \mathrm{~mL}$. The bleeding time at each step ranged from 10 minutes to 15 minutes depending on animal weight. Sequential hemodynamic changes related to the amount of blood volume removed as well as with SNP administration are shown in Figure 2. The SNP doses necessary to reach predefined MAP goals ranged from $22 \mu \mathrm{g} / \mathrm{kg} / \mathrm{min}$ to 56 $\mu \mathrm{g} / \mathrm{kg} / \mathrm{min}$. Six animals died during the experimental procedures.

Mixed ANOVA results indicated a significant effect in MAP at every steps of the experiment, but there were no differences between groups, in a marginal non-significant level $\left(\mathrm{F}=2.662, \mathrm{p}=0.053\right.$, partial eta $\left.{ }^{2}=0.27\right)$. Hence, hemorrhage and SNP groups had similar MAP values at each step of the procedure. The same procedure was conducted to analyze HR, PPV and SPV. HR was different between groups $(p<0.001)$, but PPV $(p=0.71)$ or SPV $(p=0.31)$ did not show any differences.

Although there are no MAP differences between the groups, this parameter presented earlier decreases during SNP infusion $(\mathrm{T} 1, \mathrm{p}<0.03)$ compared to the decrease observed in the hemorrhagic shock model $(\mathrm{T} 2, \mathrm{p}<0.03)$. PPV changed significantly in the two groups at $\mathrm{T} 3$ when compared to the $\mathrm{BL}$ values $(\mathrm{p}<0.05)$. On the other hand, SPV was significantly reduced at T1 $(p<0.04)$ in the SNP group, while the reduction became significant at $\mathrm{T} 4$ in the hemorrhage group $(\mathrm{p}<0.04)$. HR increased at T3 during SNP administration $(\mathrm{p}<0.02)$. HR remained unchanged during all stages of blood withdrawal $(\mathrm{p}$ $=0.12$ ), while significant changes were observed in the SNP group at $\mathrm{T} 2(\mathrm{p}<0.03)$.

\section{DISCUSSION}

In the present study, we compare the implications of two shock models (hemorrhagic shock and pharmacologic vasodilation) on arterial pressure traces. We observed that PPV was similar during all steps in both shock models. These results show that exclusive vasodilation (relative hypovolemia) can be confused with real hypovolemic states in clinical settings, especially when deliberate hypotension is necessary. Aortic pulse pressure is directly proportional to $\mathrm{SV}$ and is reflected in the peripheral pulse pressure. At the same time, respiratory PPV are inversely proportional to SV and cardiac output (CO). This dependence between pulse pressure and SV explains the PPV amplification observed in hypovolemic status. ${ }^{16,17}$ During pharmacological hypotension, SV usually remains normal or reduced, while 
vascular resistance is low. ${ }^{18,19}$ Considering the strong relation between PPV and SV, we expected that PPV would be reduced in the SNP group. However, our findings show similar increments of PPV in both the hypovolemic and pharmacological hypotension models.
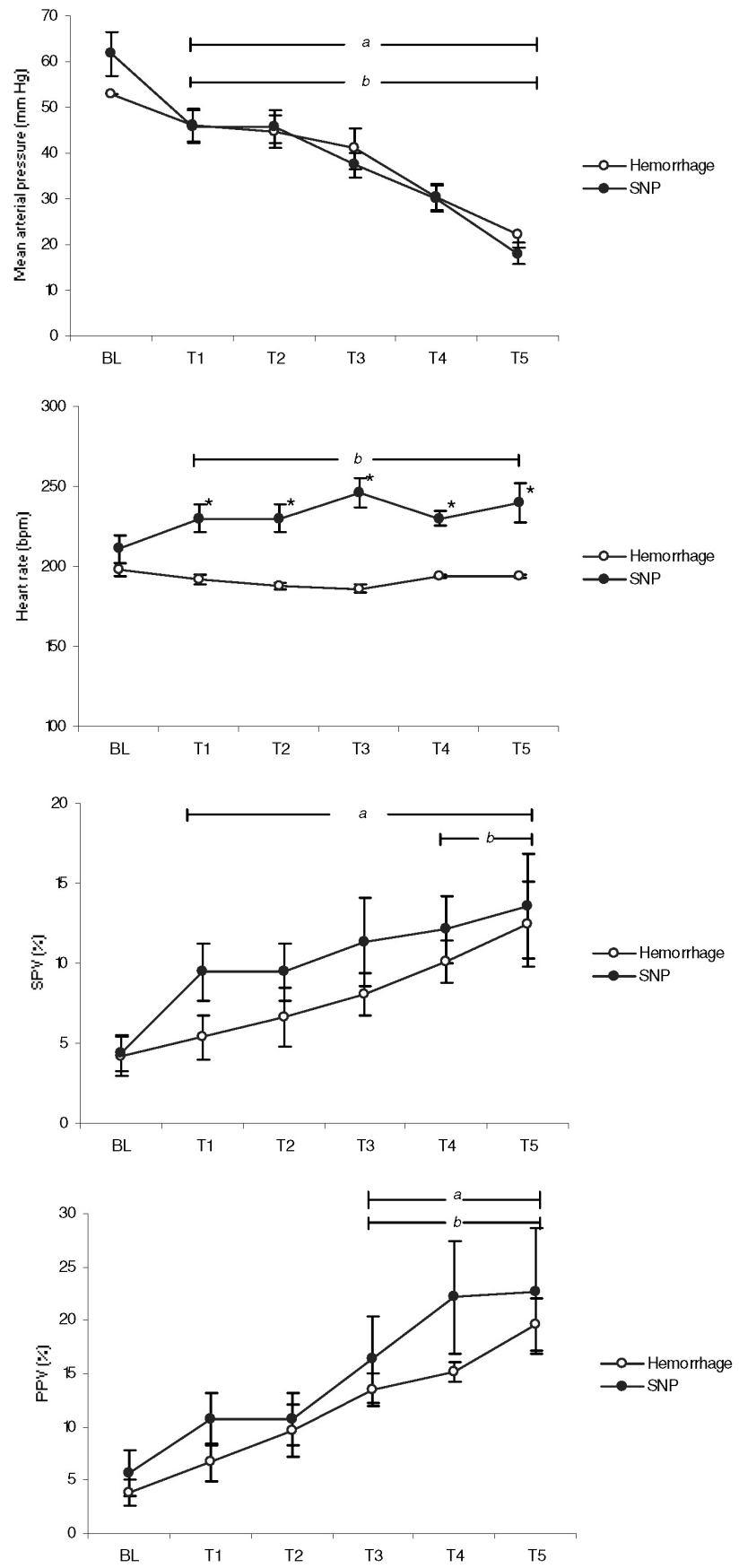

Figure 2 - Hemodynamic variables measured during experiment steps in the hemorrhage and nitroprusside (SNP) groups. Steps T1, T2, T3, T4 and T5 correspond to $10 \%, 20 \%, 30 \%, 40 \%$ and $50 \%$ of blood removal from estimated volemic state (hemorrhage group). In the SNP group, mean arterial pressure (MAP) achieved corresponds to the MAP observed at each bleeding step set in the hemorrhage group. Bars "a" (Hemorrhage) and "b" (SNP) correspond to $\mathrm{p}<0.05$ to baseline (Student's $t$-test). An asterisk shows statistical differences $(\mathrm{p}<0.05)$ between groups (ANOVA test).
In our model, requiring small animals, the SNP doses necessary to reach predefined MAP goals were greater than those habitually used in humans. The SNP has arterial and venous vasodilatory capacities, the latter being unpredictable as opposed to the arterial capacity, which is reflected by the arterial pressure. Through its venous action, the large SNP dosage could have increased microvascular volume redistribution, decreased the stressed venous volume and thus reduced the effective blood volume, SV and PPV. The significant HR increments observed in the SNP group, probably for $\mathrm{CO}$ compensation, reinforce the possibility of an SV drop. However, this is only a hypothesis that should be confirmed with a more appropriate model. On the other hand, there was no increase in HR in the hypovolemic group. Neural strategies underlying autonomic control may vary depending on the rate of blood loss. Hence, the rate of blood loss has an important impact on autonomic regulation during severe hemorrhage. Porter et al. observed that, depending on blood loss rate, HR may vary in the opposite direction. Another factor that may be involved in maintaining the heart rate in the hemorrhage group is the rate of suppression of autonomic nervous system activity triggered by the anesthesia. Kawase et al. observed heart rate lowering during hemorrhage and isoflurane anesthesia. ${ }^{20,21}$ On the other hand, NPS causes tachycardia, either through vasodilation or NO donation. This feature is important because high SNP doses were used, and the heart rate in the SNP group was significantly higher than the one in the hemorrhage group.

Hypotension during SNP administration is produced by simultaneous reductions of preload and afterload. ${ }^{18,19}$ Hence, the role of arterial compliance during mechanical ventilation must also be considered for this analysis. Ventricular afterload normally drops during mechanical inspiration because of the increased intrathoracic pressure (ITP). The decrease in afterload contributes to an increase in left ventricular SV, which increases systolic arterial pressure and arterial pulse pressure. ${ }^{19,22}$ SNP reduces afterload and may exacerbate the respiratory fluctuation of left ventricular SV, with a corresponding exaggeration in PPV and SPV. ${ }^{18,19,22}$ On the other hand, the adrenergic response, frequently present in hypovolemic states, probably partially reduces PPV and SPV in the hemorrhage group by increasing arterial elasticity and/or shifting blood from an unstressed to a stressed volume. This adrenergic effect on PPV and SPV was previously demonstrated with norepinephrine infusion during a hemorrhagic shock model. ${ }^{23}$ The authors observed that norepinephrine could significantly reduce the value of PPV and SPV and mask a true intravascular volume deficit. ${ }^{23}$

The present study did not detect an SPV statistical difference between vasodilation and hemorrhage. SPV behavior, such as the one that occurred with PPV, was 
slightly greater in the SNP group than in the hemorrhage group. By contrast, Pizov et al. demonstrated that, during NPS infusion in a dog model, SPV was not as strongly affected by pharmacologic hypotension as in the hemorrhagic scenario. ${ }^{18}$ These conflicting results may also be affected by an intense reduction in preload and afterload caused by the excessive SNP dosage used in the present study. Additionally, in the present study, the anesthetic procedure included inhalatory anesthesia with halothane, which could have amplified the vasodilatory properties of SNP. $^{24}$

In the SNP group, the SPV amplification was increased earlier (T1) than in the hemorrhage group (T4), contrasting with the PPV alteration that occurred at the same time in both groups (T3). The systolic and pulse pressure reduction seen during expiration reflects the expiratory reduction of SV. Conversely, systolic and pulse pressure increments observed in the inspiratory phase are proportional to the increases in SV. Besides the SV increments, the inspiratory gain observed in systolic pressure is additionally influenced by the aortic surrounding pressure, unlike PPV, which is basically conditioned by SV variations. Hence, at least a fraction of SPV results from airway pressure $\left(\mathrm{P}_{\text {aw }}\right)$ transmission to the vascular bed. In addition, when ITP is minimal during expiration, aortic compliance increases, arterial systolic pressure drops and SPV increases. ${ }^{16,17,22,25}$ This expiratory compliance increment may be exaggerated with SNP administration, and expiratory pulse pressure drops deeply, resulting in a major SPV. Hence, the earlier SPV amplification observed in the SNP group may reflect the transmitted airway pressure rather than SV.

When PPV is evaluated, systolic and diastolic blood pressures are measured simultaneously at the same inspiratory ITP and at the same expiratory ITP. The variable effect of ITP on aortic transmural pressure is therefore removed, making PPV a more reliable indicator of preload than SPV. However, we have to consider that, under vasoplegic conditions, expiratory pulse pressure is minimal as a result of increased aortic compliance without any augmentation of the surrounding aortic pressure..$^{16,22,25}$

This study allowed us to conclude that PPV amplification is similar in hypovolemia or pharmacologic vasodilation induced by sodium nitroprusside. SPV is influenced sooner by thoracic pressurization in the vasodilation model and may be affected by a stronger transmission of extra mural aortic pressure and reduced arterial compliance. Caution should be exercised before assuming that arterial dynamic evaluation is only a marker of intravascular volume status.

\section{REFERENCES}

1. Vincent JL. Issues in contemporary fluid management. Crit Care. 2000;4 Suppl 2:S1-2.

2. Kreimeier U. Pathophysiology of fluid imbalance. Crit Care. 2000; 4 Suppl 2:S3-7.

3. Krausz MM. Initial resuscitation of hemorrhagic shock. World J Emerg Surg. 2006;1:14.

4. Kern JW, Shoemaker WC. Meta-analysis of hemodynamic optimization in high-risk patients. Crit Care Med. 2002;30:1686-92.

5. Rivers E, Nguyen B, Havstad S, Ressler J, Muzzin A, Knoblich B, et al. Early goal-directed therapy in the treatment of severe sepsis and septic shock. N Engl J Med. 2001;345:1368-77.

6. Deitch DA. Multiple organ failure: pathophysiology and potential future therapy. Ann Surg. 1992;216:117-34.

7. Rady MY, Rivers EP, Nowak RM. Resuscitation of the critically ill in the ED: responses of blood pressure, heart rate, shock index, central venous oxygen saturation, and lactate. Am J Emerg Med. 1996;14:218-25.

8. Osman D, Ridel C, Ray P. Cardiac filling pressures are not appropriate to predict hemodynamic response to volume challenge. Crit Care Med. 2007;35:64-8.

9. Michard F, Teboul JL. Predicting fluid responsiveness in ICU patients: a critical analysis of the evidence. Chest. 2002;121:2000-8.
10. Michard F, Boussat S, Chemla D, Anguel N, Mercat A, Lecarpentier $\mathrm{Y}$, et al. Relation between respiratory changes in arterial pulse pressure and fluid responsiveness in septic patients with acute circulatory failure. Am J Respir Crit Care Med. 2000;162:134-8.

11. Hofer CK, Müller SM, Furrer L, Klaghofer R, Genoni M, Zollinger A. Stroke volume and pulse pressure variation for prediction of fluid responsiveness in patients undergoing off-pump coronary artery bypass Grafting. Chest. 2005;128:848-54.

12. Kramer A, Zygun D, Hawes H, Easton P, Ferland A. Pulse pressure variation predicts fluid responsiveness following coronary artery bypass surgery. Chest. 2004;126:1563-8.

13. Westphal GA; Garrido APG, Paoli de Almeida D, Rocha e Silva M, Poli-de-Figueiredo LF. Pulse pressure respiratory variation as an early marker of cardiac output fall in experimental hemorrhagic shock. Artif Organs. 2007;31:284-9.

14. Westphal GA, Silva E, Gonçalves AR, Caldeira Filho M, Poli-deFigueiredo LF. Pulse oximetry wave variation as a noninvasive tool to assess volume status in cardiac surgery. Clinics (Sao Paulo). 2009;64:337-43

15. Lopes MR, Auler Jr JOC, Michard F. Volume management in critically ill patients: new insights. Clinics (Sao Paulo). 2006;61:345-50.

16. Michard F. Changes in arterial pressure during mechanical ventilation. Anesthesiology. 2005;103:419-28. 
17. Pinsky MR. Recent advances in the clinical application of heart-lung interactions. Curr Opin Crit Care. 2002;8:26-31.

18. Pizov R, Ya'ari Y, Perel A. Systolic pressure variation is greater during hemorrhage than during sodium nitroprusside-induced hypotension in ventilated dogs. Anesth Analg. 1988;67:170-4.

19. Golparvar M, Naddafnia H, Saghaei M. Evaluating the relationship between arterial blood pressure changes and indices of pulse oxymetric plethysmography. Anesth Analg. 2002;95:1686-90.

20. Porter K, Ahlgren J, Stanley J, Hayward L. Modulation of heart rate variability during severe hemorrhage at different rates in conscious rats. Autonon Neurosci. 2009;150:53-61.

21. Kawase M, Komatsu T, Nishiwaki K, Kobayashi M, Kimura T, Shimada Y. Heart rate variability and arterial blood pressure variability show different characteristic changes during hemorrhage in isofluraneanesthetized, mechanically ventilated dogs. Anesth Analg. 2002;94:1621.
22. Pittman JAL, Ping JS, Mark JB. Arterial and central venous pressure monitoring. Int Anesthesiol Clin. 2004;42:13-30.

23. Nouira S, Elatrous S, Dimassi S, Besbes L, Boukef R, Mohamed B, et al. Effects of norepinephrine on static and dynamic preload indicators in experimental hemorrhagic shock. Crit Care Med. 2005;33:2339-43.

24. Harkin CP, Hudetz AG, Schmeling WT, Kampine JP, Farber NE. Halothane-induced dilatation of intraparenchymal arterioles in rat brain slices: A comparison to sodium nitroprusside. Anesthesiology. 1997;86:885-94.

25. Parry-Jones AJD, Pittman JAL. Arterial pressure and stroke volume variability as measurements for cardiovascular optimisation. Int $\mathrm{J}$ Intensive Care. 2003;10:67-72. 